\title{
Post Neurosurgical Meningitis due to Colistin Heteroresistant Acinetobacter baumannii
}

\author{
Mojtaba Moosavian ${ }^{1}$; Saeed Shoja ${ }^{1,2,}$; Roohangiz Nashibi ${ }^{3}$; Nasim Ebrahimi ${ }^{1}$; Mohammad \\ Amin Tabatabaiefar ${ }^{4}$; Soodabeh Rostami ${ }^{1}$; Amir Peymani $^{5}$ \\ ${ }^{1}$ Microbiology Department, Infectious and Tropical Diseases Research Center, Ahvaz Jundishapur University of Medical Sciences, Ahvaz, IR Iran \\ ${ }^{2}$ Infectious and Tropical Diseases Research Center, Hormozgan University of Medical Sciences, Bandar Abbas, IR Iran \\ ${ }_{3}^{3}$ Infectious and Tropical Diseases Research Center, Razi Hospital, Infectious and Tropical Disease Ward, Ahvaz Jundishapur University of Medical Sciences, Ahvaz, IR Iran \\ ${ }_{5}^{4}$ Genetic Department, Ahvaz Jundishapur University of Medical Sciences, Ahvaz, IR Iran \\ ${ }_{5}$ Microbiology Department, Qazvin University of Medical Sciences, Qazvin, IR Iran \\ ${ }^{*}$ Corresponding author: Saeed Shoja, Microbiology Department, Infectious and Tropical Diseases Research Center, Ahvaz Jundishapur University of Medical Sciences, Ahvaz, IR Iran. \\ Tel: +98-6113367543, Fax:+98-6113332036, E-mail: shojasaeed@gmail.com
}

Received: May 18, 2013; Revised: September 22, 2013; Accepted: October 20, 2013

\begin{abstract}
Introduction: Recently Acinetobacter baumannii isolates have emerged as a problematic infectious agent that causes meningitis in neurosurgical patients. Colistin has been used successfully for the treatment of A. baumannii meningitis but colistin resistant isolates have been reported worldwide.

Case Presentation: Two isolates of A. baumannii were cultured during a five-day period from cerebrospinal fluid (CSF) samples of a 20-year-old man with a gunshot trauma in the abdomen, which had exited from his back. Antimicrobial susceptibility tests of isolates were performed. Multiplex PCR was performed for detection of $b l a_{\text {OXA-23-like, }}, b l a_{\text {OXA-24-like }}$ and $b l a_{\text {OXA-58-like }}$ genes. Metallo- $\beta$-lactamase genes such as: $b l a_{\mathrm{VIM}}, b l a_{\mathrm{IMP}}, b l a_{\mathrm{SPM}}$ and $b l a_{\mathrm{NDM}}$ were sought by singleplex PCR. In order to evaluate the genetic relationship, two isolates were examined by the repetitive extragenic palindromic-polymerase chain reaction (REP_PCR) method.

Conclusions: E-test results showed that the isolates were sensitive to colistin and tigecycline with minimum inhibitory concentration of (MIC) $0.25 \mu \mathrm{g} / \mathrm{mL}$ and $1.5 \mu \mathrm{g} / \mathrm{mL}$, respectively. Secondly the isolates were resistant to colistin with MIC $>256 \mu \mathrm{g} / \mathrm{mL}$ but remained sensitive to tigecycline with MIC $1.5 \mu \mathrm{g} / \mathrm{mL}$. On the basis of the multiplex PCR, both of the isolates were positive for bla $\mathrm{OXA}_{\text {-23-like }}$. Other investigated genes such as $b l a_{\mathrm{OXA}-24-\mathrm{like}}, b l a_{\mathrm{OXA}-58-\mathrm{like}}, b l a_{\mathrm{VIM}}, b l a_{\mathrm{IMP}}, b l a_{\mathrm{SPM}}$ and $b l a_{\mathrm{NDM}}$ were negative. REP-PCR results showed that two isolates were derived from a single strain and both were the same. The results of our study revealed that the firs isolate of A. baumannii was colistin heteroresistant and was changed to completely resistant during therapy. Diagnosis and treatment of A. baumannii meningitis is very important and to avoid treatment failure we suggest that all $A$. baumannii isolates obtained from CSF should be evaluated properly for colistin heteroresistance.
\end{abstract}

Keywords:Acinetobacter baumannii; Meningitis; Colistin; Neurosurgery

\section{Introduction}

Acinetobacter baumannii has become an important pathogen that causes nosocomial infections such as bloodstream and wound infections, ventilator-associated pneumonia and meningitis (1). Approximately 10\% of Gram-negative and $4 \%$ of nosocomial meningitis occur as a result of A. baumannii infection (2). Neurosurgical patients with cerebrospinal fluid (CSF) leakage have a high risk of acquiring meningitis. A. baumannii is a serious pathogen that causes meningitis in these patients (3) and many authors worldwide have reported post neurosurgical meningitis due to A. baumannii (4). Since A. baumannii strains are multidrug resistant, nosocomial infections associated with neurosurgical procedures such as nosocomial meningitis caused by $A$. baumannii had a high rate of mortality $(5,6)$; two studies have reported a mortality rate ranging from $71.4 \%$ to $72.7 \%$, respectively for $A$. baumannii meningitis in neurosurgical patients $(5,7)$.

In the recent years, as a result of excessive use of an- tibiotics, the epidemiology of post-neurosurgical meningitis may have changed, and because treatment options for multidrug resistant A. baumannii are limited, the rate of meningitis due to this bacterium will be increased (2). Colistin is the last resort for treatment of pan drug-resistant $A$. baumannii, but increased use of this antibiotic has led to progress of colistin-resistant strains (1). The first colistin heteroresistance in A. baumannii was described in 2006. This type of resistance may be the primary stage that upon contact with colistin, resistant subpopulations can proliferate (8). Colistin heteroresistance seriously warns that colistin should be used properly. If colistin is used improperly, resistance may develop and lead to treatment failure (9). We report a case of post-neurosurgical meningitis due to colistin heteroresistant A. baumannii, with some changes in antibiotic susceptibility profile during intratreatment with colistin.

Copyright (C 2014, Ahvaz Jundishapur University of Medical Sciences; Published by Kowsar Corp. This is an open-access article distributed under the terms of the Creative Commons Attribution License, which permits unrestricted use, distribution, and reproduction in any medium, provided the original work is properly cited. 


\section{Case Presentation}

Our case was a 20-year-old man who had a gunshot trauma to the abdomen, which had exited from his back. The patient was admitted and underwent laparotomy three times. There was a debrided ulcer on his back with leakage of CSF. One week after his discharge, the patient became febrile $\left(\mathrm{T} \geq 39^{\circ} \mathrm{C}\right.$ ) and was admitted to our unit with a diagnosis of meningitis and treated with meropenem, vancomycin and amikacine. Cerebrospinal fluid analysis was as follows: total cell count $=230$, total white blood cells $(\mathrm{WBC})=200$, polymorph nuclear $(\mathrm{PMN})=70 \%$, lymphocytes $(\mathrm{LYM})=30 \%$, glucose $39 \mathrm{mg} / \mathrm{dl}$ and protein $40 \mathrm{mg} /$ dL. The radiological study revealed hydrocephalus, therefore extra ventricular drainage (EVD) was implanted. After four days, samples from the ulcer, urine and CSF yielded A. baumannii. Finally the patient was referred to our center (Golestan Hospital, Ahvaz, southwest of Iran). At this center, EVD was revised twice and the patient received antibiotic therapy with ampicillin/sulbactam, piperacillin/ tazobactam, rifampin and ciprofloxacin in different combinations.

On the day of the admission a CSF sample was taken and cultured. After growth, colistin sensitive A. baumannii (isolate number 1) was recovered. After four days, colistin (intravenous and intrathecal) was initiated. Five days after administration of colistin, another CSF sample was taken and cultured again, and colistin resistant A. baumannii (isolate number 2) was recovered. The patient was still ill and did not improve. On the thirteenth day of treatment with colistin, a CSF sample was taken again, analyzed and cultured. However, the culture was negative for A. baumannii but CSF analysis showed meningitis pattern that was as follows: total cell count $=11600$, total white blood cells $(\mathrm{WBC})=1600$, polymorph nuclear $(\mathrm{PMN})=85 \%$, Lymphocytes (LYM) $=15 \%$, glucose $25 \mathrm{mg} / \mathrm{dL}$ and protein $65 \mathrm{mg} / \mathrm{dL}$. Colistin treatment was continued until the last day of his life. The patients expired after 26 days of hospitalization. According to the importance of diagnosis and treatment of A. baumannii meningitis, and since this case of meningitis was particular and rare, we decided to do some additional experiments on two isolates of the A. baumannii.

Conventional microbiology tests were performed for initial identification of A. baumannii (10). DNA was extracted by sodium dodecyl sulfate (SDS) and the proteinase K method (11) and, bla ${ }_{\mathrm{OXA}-51-l i k e}$ gene and partial sequencing of $r p o B$ gene were performed by specific primers (12, 13). A. baumannii NCTC 12156 (ATCC 19606) was used as the positive control (14). Antimicrobial susceptibility testing of the two isolates to the following antibiotics was evaluated by the disk diffusion method (15). The tested antibiotics were as follow: imipenem 10 $\mu \mathrm{g}$, meropenem $10 \mu \mathrm{g}$, ceftazidime $30 \mu \mathrm{g}$, cefepime 30 $\mu \mathrm{g}$, ceftriaxone $30 \mu \mathrm{g}$, colistin $10 \mu \mathrm{g}$, piperacillin $100 \mu \mathrm{g}$, piperacillin-tazobactam 100/10 $\mu \mathrm{g}$, polymyxin B 300 unit, gentamicin $10 \mu \mathrm{g}$, tobramycin $10 \mu \mathrm{g}$, amikacin 30 $\mu \mathrm{g}$, tetracycline $30 \mu \mathrm{g}$, ciprofloxacin $5 \mu \mathrm{g}$, trimethoprimsulfamethoxazole 1.25/23.75 $\mu$, rifampin $5 \mu$ g, aztreonam $30 \mu \mathrm{g}$ and ampicillin-sulbactam $(10 / 10 \mu \mathrm{g})$ (MAST, Group Ltd, Merseyside, UK). Minimum inhibitory concentration (MIC) of colistin, imipenem, meropenem and tigecycline were determined by the E-test (Liofilchem, Italy). The results of disk diffusion and E-test were interpreted according to CLSI guidelines. The United States Food and Drug Administration-approved criteria for Enterobacteriaceae was used for the tigecycline breakpoint (16).

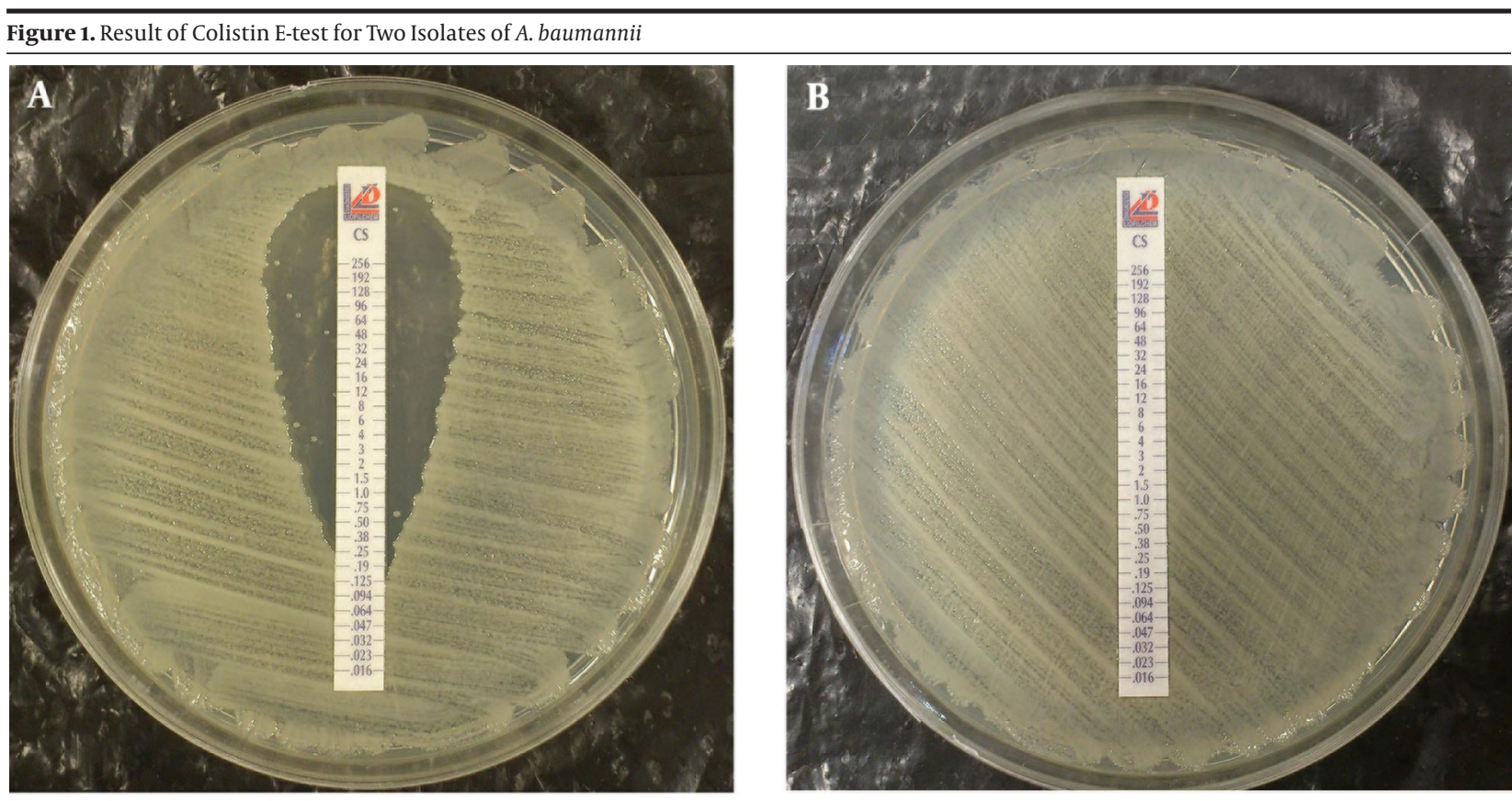

A, Result of colistin E-test on the first isolate of A. baumannii; Eight colonies grew within the zone of inhibition. B, Result of colistin E-test on second isolate of A. baumannii recovered after colistin therapy. 


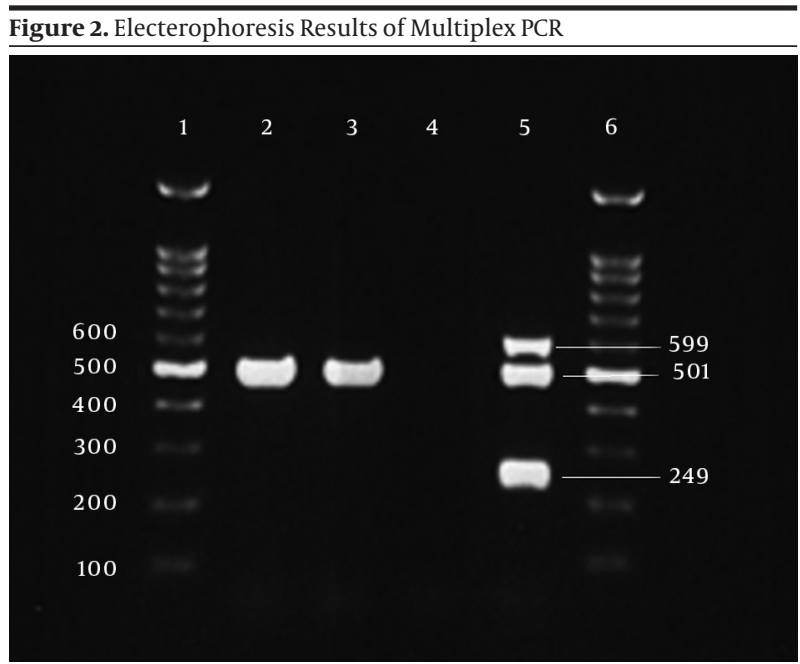

Lanes 1 and 6,100 bp DNA ladder; Lane 2, first isolate with bla ${ }_{\text {OXA23-like }}$ gene at 501 bp; Lane 3, second isolate with bla OXA23-like gene; Lane 4, negative control; lane 5, positive control, bla OXA24-like at 249 bp, bla OXA23like ${ }^{\text {at } 501 \text { bp and bla }}$ OXA58-like ${ }^{\text {at } 599 \text { bp. }}$

Multiplex PCR for detection of oxacillinase genes such as: blaOXA23-like, blaOXA-24-like and blaOXA-58-like were performed as previously described (14). Metallo ß-lactamase production and encoding genes such as: blaIMP, blaVIM, blaSPM and blaNDM were investigated by PCR(17, 18). Both isolates were examined by REP-PCR with primers according to the Bou et al. protocol with some modifications (11). According to the PCR results, both isolates were $b a_{\text {OXA-51-like }}$ positive and according to sequences of rpoB gene alignment, both isolates were confirmed as $A$. baumannii. Isolate 1 was resistant to all of tested antimicrobial disks except polymyxin B, colistin and ampicillinsulbactam. Results of E-test showed that isolate 1 was resistant to imipenem and meropenem with MIC $>32 \mu \mathrm{g} /$ $\mathrm{mL}$ and $12 \mu \mathrm{g} / \mathrm{mL}$, respectively, yet was sensitive to colistin with MIC $0.25 \mu \mathrm{g} / \mathrm{mL}$. Although disk diffusion results showed that isolate 1 was resistant to tigecycline yet the E-test result revealed tigecycline MIC $1.5 \mu \mathrm{g} / \mathrm{mL}$, and isolate 1 was categorized as sensitive to tigecycline according to the E-test result. However isolate 1 was sensitive to colistin, but within the colistin zone of inhibition, eight colonies grew, which are indicated in Figure 1-A.

Antibiogram results of the second isolate that was recovered after colistin therapy was similar to the first isolate but some changes had occurred. The second isolate was completely resistant to colistin with MIC $256 \mu \mathrm{g} / \mathrm{mL}$ (Figure 1-B). By disk diffusion, isolate 2 was resistant to both of polymyxin $\mathrm{B}$ and colistin but remained sensitive to ampicillin-sulbactam. The MIC results for imipenem, meropenem and tigecycline were unchanged for the second isolate. Multiplex PCR revealed that two isolates had the blaOXA23-like gene (Figure 2). Other investigated genes were negative for both isolates. By phenotypic tests, metallo-ß-lactamase production was negative for the two isolates. Results of REP-PCR revealed that the two isolates were derived from a single strain and were same (Figure 3).

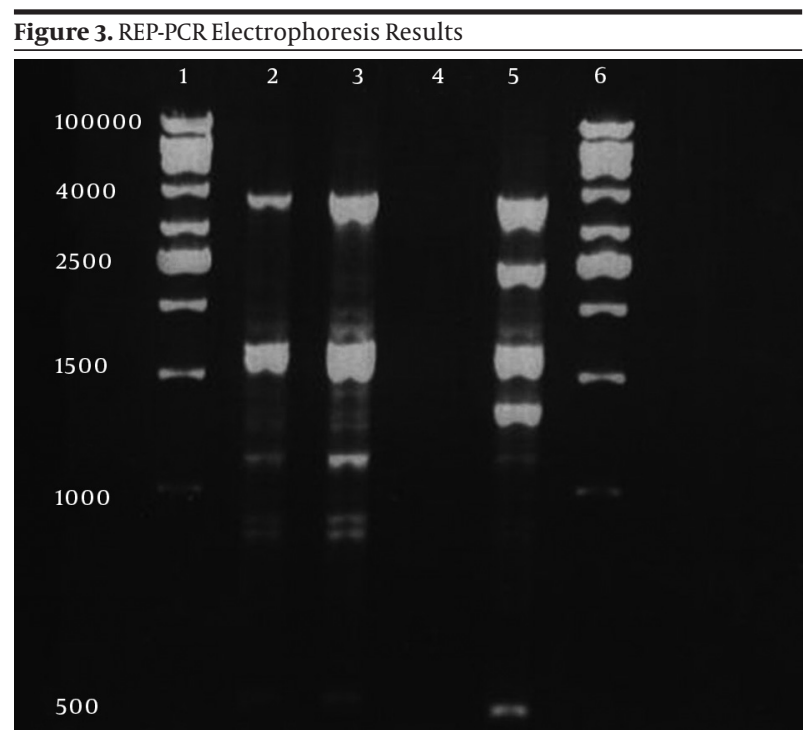

Lanes 1 and 6, 1kb DNA ladder; Lane 2, first isolate; Lane 3, second isolate; Lane 4, negative control and Lane 5, A. baumannii ATCC 19606.

\section{Discussion}

Nosocomial meningitis due to A. baumannii is fatal and its treatment is difficult (19). Recently, post neurosurgical meningitis caused by colistin heteroresistant A. bauman$n i i$ has been reported $(20,21)$. Due to difficulties in detection, the prevalence of colistin heteroresistance is not yet well understood and is not routinely used in most laboratories $(9,21)$ but Overall, the colistin heteroresistance rate in A. baumannii was much higher than the resistance rate (9). In the present study at the beginning of colistin treatment, isolate 1 was susceptible to colistin with MIC 0.25 $\mu \mathrm{g} / \mathrm{mL}$ but some colonies were grown within the zone of inhibition that indicate heteroresistant subpopulations were present. Five days after treatment with colistin, isolate 2 was recovered with colistin MIC $>256 \mu \mathrm{g} / \mathrm{mL}$ that was completely resistant to colistin. It seems that during intra-treatment in vivo, a colistin resistant subpopulation was selected. One similar finding was reported by Rodriguez et al. from Argentina (21).

The results of our study is in contrast to that of Rodriguez et al. because in our study we were not able to treat meningitis but Rodriguez et al. successfully treated the meningitis due to A. baumannii. The difference between the results of the two studies could be due to the susceptibility pattern of the two isolates to rifampin, because our isolates were resistant to rifampin but in the Rodriguez et al. study the A. baumannii isolates were susceptible and responded to rifampin and colistin therapy. Moreover, successful treatment of post-neurosurgical meningitis caused by multidrug-resistant $A$. baumannii with intrathecal colistin has been previously reported (2) but unfortunately in this study, colistin was not effective even through intrathecal use, because the first isolate was heteroresistant and became completely resistant during the treatment. However, previous exposure to colistin may 
be a risk factor for development of heteroresistance (9) yet in this patient, no treatment with colistin was performed previously.

In vitro synergy between rifampin and colistin has been shown previously (20). Rifampin has an excellent CSF penetration and was used for the treatment of meningitis caused by A. baumannii $(2,22)$. Unfortunately, our isolates in addition to colistin were resistant to rifampin thus, although we used a combination of two drugs (colistin and rifampin) treatment was not effective. The two isolates were sensitive to ampicillin-sulbactam in vitro and we used this antibiotic for treatment, but the patient did not respond to this antibiotic either. Tigecycline has in vitro activity on pan-drug resistant acinetobacter and some studies reported successful treatment of meningitis caused by multidrug resistant $A$. baumannii with tigecycline, yet this treatment option is very expensive and is not easily available in developing countries $(23,24)$. Moreover, our isolates were susceptible to tigecycline in vitro, but tigecycline is not available in Iran. Interestingly we observed a strange phenomenon regarding polymyxin B. The first isolate of A. baumannii was susceptible to polymyxin $B$ by disk diffusion, but the second isolate was completely resistant to polymyxin B without any previous exposure to this antibiotic, because polymyxin B was not use in the treatment of the patient. We could not find any reason for this phenomenon.

It is assumed that because colistin and polymyxin B belong to one family, cross-resistance may occur between them. In comparison to the first isolate, in the second isolate other antibiotics MIC, and disk diffusion profile, were not changed intratreatment and the second isolate remained sensitive to ampicillin-sulbactam and tigecycline in vitro. For detection of carbapenemase genes we investigated class D, OXA genes that are the most active for resistance to carbapenems in A. baumannii (25). We found that two isolates had blaOXA23-like gene. Other investigated genes were negative in both isolates. To confirm that both isolates were from identical sources and that the patient was not infected with other A. baumannii strains during his stay in the intensive care unit (ICU), REP-PCR was performed according to the Bou et al., protocol (11). Results of REP-PCR revealed that the two isolates were derived from a single strain. This case report is unique because generation of polymyxin resistant $A$. baumannii during colistin therapy has not been reported previously. In the present study, the disk diffusion method failed to detect colistin heteroresistance subpopulation in the first isolate and we recommend, in isolates that were recovered for the first time from CSF or were recovered during intratreatment, colistin MIC should be measured with E-test or other MIC methods.

\section{Acknowledgements}

We acknowledge the Research Consultation Center (RCC) of vice-chancellor of Ahvaz Jundishapur University of Medical Sciences for their technical support.

\section{Authors' Contributions}

Study concept and design: Mojtaba Moosavian, Saeed Shoja and Roohangiz Nashibi. Acquisition of data: Saeed Shoja. Analysis and interpretation of data: Saeed Shoja, Soodabeh Rostami and Nasim Ebrahimi. Drafting of the manuscript: Saeed Shoja, Mojtaba Moosavian and Roohangiz Nashibi. Critical revision of the manuscript for important intellectual content: Mohammad Amin Tabatabaiefar and Amir Peymani. Statistical analysis: Saeed shoja and Soodabeh Rostami. Study supervision: Mojtaba Moosavian.

\section{Financial Disclosure}

This study was part of a PhD thesis, granted an approved by plan number 90126 and was financially supported by the deputy vice-chancellor for research affairs of Ahvaz Jundishapur University of Medical Sciences, and Infectious and Tropical Disease Research Center.

\section{Funding/Support}

Funding for this work was provided by the deputy vicechancellor for research affairs of Ahvaz Jundishapur University of Medical Sciences, and Infectious and Tropical Disease Research Center (Grant Number 90126).

\section{References}

1. Kempf M, Rolain JM. Emergence of resistance to carbapenems in Acinetobacter baumannii in Europe: clinical impact and therapeutic options. Int J Antimicrob Agents. 2012;39(2):105-14

2. Cascio A, Conti A, Sinardi L, Iaria C, Angileri FF, Stassi G, et al. Post-neurosurgical multidrug-resistant Acinetobacter baumannii meningitis successfully treated with intrathecal colistin. A new case and a systematic review of the literature. Int I Infect Dis. 2010;14(7):e572-9.

3. Kim BN, Peleg AY, Lodise TP, Lipman J, Li J, Nation R, et al. Management of meningitis due to antibiotic-resistant Acinetobacter species. Lancet Infect Dis. 2009;9(4):245-55.

4. Khan FY, Abukhattab M, Baager K. Nosocomial postneurosurgical Acinetobacter baumannii meningitis: a retrospective study of six cases admitted to Hamad General Hospital, Qatar. J Hosp Infect. 2012;80(2):176-9.

5. Tuon FF, Penteado-Filho SR, Amarante D, Andrade MA, Borba LA. Mortality rate in patients with nosocomial Acinetobacter meningitis from a Brazilian hospital. Braz J Infect Dis. 2010;14(5):437-40.

6. Paramythiotou E, Karakitsos D, Aggelopoulou H, Sioutos P, Samonis G, Karabinis A. Post-surgical meningitis due to multiresistant Acinetobacter baumannii. Effective treatment with intravenous and/or intraventricular colistin and therapeutic dilemmas. Med Mal Infect. 2007;37(2):124-5.

7. Metan G, Alp E, Aygen B, Sumerkan B. Acinetobacter baumannii meningitis in post-neurosurgical patients: clinical outcome and impact of carbapenem resistance. J Antimicrob Chemother 2007;60(1):197-9.

8. Li J, Rayner CR, Nation RL, Owen RJ, Spelman D, Tan KE, et al. Heteroresistance to colistin in multidrug-resistant Acinetobacter baumannii. Antimicrob Agents Chemother. 2006;50(9):2946-50.

9. Cai Y, Chai D, Wang R, Liang B, Bai N. Colistin resistance of Acinetobacter baumannii: clinical reports, mechanisms and antimicrobial strategies. J Antimicrob Chemother. 2012;67(7):1607-15.

10. Winn WC, Allen SD, Janda WM, Koneman EW, Schreckenberger PC, Procop GW, et al. Koneman's Color Atlas and Textbook of Diagnostic microbiology. 16 th ed: Williams \& Wilkins; 2006.

11. Bou G, Cervero G, Dominguez MA, Quereda C, Martinez-Beltran 


\section{Moosavian $M$ et al.}

J. PCR-based DNA fingerprinting (REP-PCR, AP-PCR) and pulsedfield gel electrophoresis characterization of a nosocomial outbreak caused by imipenem- and meropenem-resistant Acinetobacter baumannii. Clin Microbiol Infect. 2000;6(12):635-43.

12. Turton JF, Woodford N, Glover J, Yarde S, Kaufmann ME, Pitt TL Identification of Acinetobacter baumannii by detection of the blaOXA-51-like carbapenemase gene intrinsic to this species. $J$ Clin Microbiol. 2006;44(8):2974-6.

13. Gundi VA, Dijkshoorn L, Burignat S, Raoult D, La Scola B. Validation of partial rpoB gene sequence analysis for the identification of clinically important and emerging Acinetobacter species. Microbiology. 2009;155(Pt 7):2333-41.

14. Woodford N, Ellington MJ, Coelho JM, Turton JF, Ward ME, Brown S, et al. Multiplex PCR for genes encoding prevalent OXA carbapenemases in Acinetobacter spp. Int J Antimicrob Agents. 2006;27(4):351-3.

15. Pentecostal Assemblies of the World. M 100-S20 Performance standards for antimicrobial susceptibility testing: 20 th informational supplement. Clinical and Laboratory Standards Institute. 2011.

16. Wyeth Pharmaceuticals. TYGACIL (tigecycline) injection, powder, lyophilized, for solution. Philadelphia; 2005.

17. Zong Z, Lu X, Valenzuela JK, Partridge SR, Iredell J. An outbreak of carbapenem-resistant Acinetobacter baumannii producing OXA-23 carbapenemase in western China. Int J Antimicrob Agents. 2008;31(1):50-4.

18. Poirel L, Walsh TR, Cuvillier V, Nordmann P. Multiplex PCR for detection of acquired carbapenemase genes. Diagn Microbiol Infect
Dis. 2011;70(1):119-23.

19. Krol V, Hamid NS, Cunha BA. Neurosurgically related nosocomial Acinetobacter baumannii meningitis: report of two cases and literature review.J Hosp Infect. 2009;71(2):176-80.

20. Rodriguez CH, Barberis C, Nastro M, Bombicino K, Granados G Vay $C$, et al. Impact of heteroresistance to colistin in meningitis caused by Acinetobacter baumannii. J Infect. 2012;64(1):119-21.

21. Rodriguez CH, Bombicino K, Granados G, Nastro M, Vay C, Famiglietti A. Selection of colistin-resistant Acinetobacter baumannii isolates in postneurosurgical meningitis in an intensive care unit with high presence of heteroresistance to colistin. Diagn Microbiol Infect Dis. 2009;65(2):188-91.

22. Motaouakkil S, Charra B, Hachimi A, Nejmi H, Benslama A, Elmdaghri N, et al. Colistin and rifampicin in the treatment of nosocomial infections from multiresistant Acinetobacter baumannii. J Infect. 2006;53(4):274-8.

23. Saleem AF, Shah MS, Shaikh AS, Mir F, Zaidi AK. Acinetobacter species meningitis in children: a case series from Karachi, Pakistan. J Infect Dev Ctries. 2011;5(11):809-14.

24. Tutuncu EE, Kuscu F, Gurbuz Y, Ozturk B, Haykir A, Sencan I. Tigecycline use in two cases with multidrug-resistant Acinetobacter baumannii meningitis. Int JInfect Dis. 2010;14 Suppl 3:e224-6.

25. D'Arezzo S, Principe L, Capone A, Petrosillo N, Petrucca A, Visca P. Changing carbapenemase gene pattern in an epidemic multidrug-resistant Acinetobacter baumannii lineage causing multiple outbreaks in central Italy. J Antimicrob Chemother. 2011;66(1):54-61. 